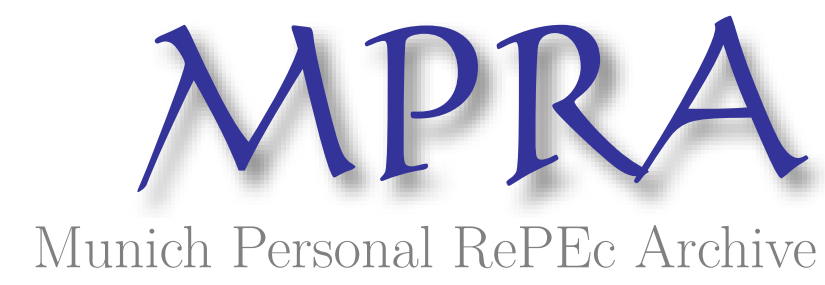

\title{
Choosy search and the mismatch of talents
}

Decreuse, Bruno

GREQAM

2003

Online at https://mpra.ub.uni-muenchen.de/3636/

MPRA Paper No. 3636, posted 20 Jun 2007 UTC 


\title{
Choosy search and the mismatch of talents*
}

\author{
Bruno Decreuse ${ }^{\dagger}$ \\ GREQAM, Université de la Méditerranée
}

\begin{abstract}
This paper proposes a multi-sector matching model where workers have (symmetric) sector-specific skills and the search market is segmented by sector. Workers choose the range of markets they are willing to participate in. I identify a composition externality: workers do not take into account the impact of their choice on sector-specific mean productivity among the pools of job-seekers. Consequently, workers prospect too many market segments, and there is room for public policy even when the so-called Hosios condition holds.
\end{abstract}

Keywords: Composition effects; Heterogeneity; Segmented markets; Efficiency

J.E.L. classification: J64, J65, D33

*This paper has benefited from discussions with Yann Algan, Pierre Cahuc, Olivier Charlot, François Fontaine, Cecilia Garcia-Penalosa, Pierre Granier, Michel Normandin and Bertrand Wigniolle. I also wish to thank seminar participants at Université Paris I, Université du Maine, Université Cergy-Pontoise, Université de la Méditerranée, participants to the EEA-ESEM conference in Stockholm, and the T2M conference in Orléans, as well as two referees and an editor of this review who considerably helped to improve the content of the paper. The usual disclaimer applies.

†GREQAM, - Vieille Charité, 2 rue de la charité, 13236 Marseille cedex 02, France. Phone: 33-49114-0721. E-mail: decreuse@univmed.fr 


\section{Introduction}

This paper examines the efficiency of workers' decision to search. Searching for a job involves deciding how much to search. This gives rise to important externalities, since it modifies the matching opportunities of other agents: when one individual engages in search, she raises the probability that firms fill their vacancies, but creates congestion which reduces the rate at which other job seekers find a job. It has been shown that those two externalities can offset each other in particular cases (see Mortensen, 1982, and Hosios, 1990). In this paper, I complement this analysis by considering a matching model where workers have multi-dimensional skills. In this framework, searching for a job involves deciding how far to search. Hence, the focus is on the trade-off made by the unemployed between complete specialization and the odds of employment. Matching externalities then result in an inefficient allocation of workers to jobs. I show that the unemployed are insufficiently selective, i.e. they prospect too many jobs.

The main motivation of our work relates to the impact of heterogeneity on efficiency in frictional models of the labour market. Two main papers have addressed this question, and they obtained very different results. On the one hand, Marimon and Zilibotti (1999) hereafter MZ - offer the 'mismatch of talents' framework. This is a multi-sector matching model with ex-post Nash bargaining over the match surplus where workers have (symmetric) sector-specific skills à la Salop (1979). Firms and workers are distributed along the perimeter of a technological circle. The productivity of a pair is strictly decreasing in the distance between firm's and worker's locations. Search is random: any worker may meet any firm. The typical problem faced by a firm and a worker at the recruitment stage is whether to form a match or not. MZ show that the Hosios condition - that is, workers' bargaining power must be equal to the elasticity of the matching function with respect to the pool of unemployed - decentralizes the efficient allocation. On the other hand, Shimer and Smith (2001) - hereafter SS - point out a major source of inefficiency. In their framework, workers differ in skill levels, choose the magnitude of their search investment, and can accept or reject a job offer. They show that skilled workers undertake too low search investments, and reject too few offers. Conversely, unskilled workers undertake a too high search intensity, and reject too many offers. Put otherwise, the efficient outcome cannot be decentralized.

The purpose of this paper is to understand these two conflicting results better by revisiting the MZ model. I proceed in two steps. First, I slightly alter the MZ model and show that the decentralized outcome cannot be efficient in that modified model. Second, I compare this model formally to MZ and SS.

Concerning the first step, I assume that the search market is fully and endogenously segmented by location. On each (sub-)market, job-seekers and vacancies meet according to an (exogenous) constant returns to scale matching technology. The supply of vacancies in each location is determined by a free entry condition. I assume that workers select the range of markets in which they want to participate. Indeed, sending the worker where he is best suited for does not insure he will be hired. The choice of subset is made on the basis of a reservation product strategy, i.e. only segments that may offer a wage above the reservation wage are prospected. There exists an exogenous cost of search which is arbitrarily small (or alternatively, individuals have a disutility if rejected for a job) which 
implies that workers do not apply for jobs they are not willing to occupy. To distinguish this case from MZ, I refer to this search process as choosy search.

Unlike random search, choosy search generates a composition externality. While choosing whether to enter a sub-market or not, workers do not take into account the impact their participation has on the composition of the pool of job-seekers. This composition in turn affects firms' willingness to advertise vacancies. As a result, there is no value of workers' bargaining power that can decentralize the efficient allocation. In addition, I find workers always search on a too wide range of markets. Indeed, workers prospect jobs in locations that are too far from their own. From firms' point of view, it is never worth rejecting the application of these low ability workers, though the discounted output flow generated by these workers is below the average cost to recruit them. This inability to refuse matches ex-post that are ex-ante inefficient is due to the fact that the cost of advertising a vacancy is sunk at the time of hiring. No decentralized mechanism is at play to deter the participation of individuals in sectors that are not well-suited to their skills. In the absence of collective agreements to set minimum standards in the market for skills, search is not sufficiently choosy.

Concerning the second step, I slightly alter my model to compare it with MZ and SS. MZ corresponds to the case where agents search in all locations, regardless of whether they are willing to take the job or not. I introduce an error component in the choice made by the agents about the subset of locations in which they try to get a job. With some exogenous probability, agents get a random offer from the whole circle. I show that the externality vanishes as the latter probability tends to one. This explains why the efficient outcome can be decentralized in MZ: workers make systematic errors while sampling jobs, and cannot use the information they have concerning the skill requirement of jobs. As far as they can use this information, sampling becomes more selective, but insufficiently. To compare with SS, I allow the agents to set their search intensity in each location ${ }^{1}$. As agents differ in the composition of their skills, there is a continuum of skills in each location. I show that workers search too little for jobs they are highly qualified for and too much for jobs they are less qualified for. Hence, I can replicate the SS results on each location of the technological circle. Thus, the insight of this paper is that in an environment with heterogeneity in match quality, if workers can refine their search either by choosing the subset of jobs to prospect, or by setting a different search intensity for each type of job - it will typically not be possible to achieve efficiency with bargaining alone.

This paper emphasizes the fragility of the Hosios condition, when we marginally modify the way in which workers choose which jobs to apply for. This is particularly striking, since from the individual point of view, workers are actually indifferent between the two rules (choosy search vs random search) for selecting which jobs to solicit. As an analogy, suppose all unemployed workers could subscribe to a service that e-mails them about open vacancies as soon as information about these vacancies becomes available. Suppose the service could design a filter so that an unemployed worker only receives the e-mail if a vacancy fits a certain criterion. As long as a worker does not mind looking at vacancies

\footnotetext{
${ }^{1}$ The number of firms is exogenous in SS, while there is free entry in my paper. Actually, the way the model is closed is not very important. All that matters is the composition externality vehicled by search decisions.
} 
for jobs he rejects (i.e. search is costless), he would be indifferent between having such a filter installed or not; that is, he would not care whether he learns about the vacancy and is given the chance to say no as opposed to never being told about the vacancy in the first place. But the possibility of using filters matters. The intuition is as follows: if the filter allows firms to direct their vacancies to those who express an interest in the job, there will be fewer mismatches and search will be more efficient. More interestingly, a decentralized search process will fail to be efficient when we allow for such filters, even when the Hosios condition is satisfied. The reason is that workers fail to take into account that when they put down their name for a vacancy, they create congestion for others. The size of such congestion externality does not depend on workers' type, but the benefit accruing from search does. As a result, workers end up applying for too many jobs, and welfare would be higher if all workers searched more narrowly.

This paper is related to the literature that highlights a composition externality in matching environments. Such composition externality always happens with random search and vertical heterogeneity ${ }^{2}$, that is when agents differ in a single (productive) characteristic (see, e.g., Lockwood, 1986, and SS). In directed search models, the search market is usually segmented by workers' type, and workers search jobs in a single market segment. There are no mismatch as a result (see, e.g., Shi, 2001, and Shimer, 2005). However, there are models in which the search market is segmented by job type, but not by workers' type. Workers direct their search towards a particular market, but matching is random within this market. In the two-sector models of Charlot and Decreuse (2005) and Uren (2006), the participation decision thus generates a composition externality ${ }^{3}$. A key aspect of this paper is to show that the composition externality survives when we consider horizontal heterogeneity - workers have similar levels of skills, but in different fields - rather than vertical heterogeneity.

The notion of choosy search has already been used by Moscarini (2001), who proposes a two sector matching model in which the contact technology depends linearly on the number of active job-seekers, and workers may have any bundle of sector-specific skills. However, he does not perform a normative analysis. Choosy search can be compared to the notions of marketability and specialization highlighted in the literature on money and search (see e.g. Kiyotaki and Wright, 1993, and Shi, 1997). The main idea in these papers is that each producer faces a trade-off between specialization and marketability. Specializing in the production of a given commodity allows better productivity (or, equivalently, saves on production costs), but at the expense of reducing the proportion of consumers interested in purchasing the good, i.e. marketability is smaller. Typically, money plays a crucial role in this approach as it enlarges the size of the market and therefore allows producers to specialize. Under choosy search, there is no restriction on the number of sub-markets that each worker may prospect, but the requirement that expected matching

\footnotetext{
${ }^{2}$ In Gautier and Teuling (2004), workers differ in a skill level, while jobs differ in a complexity level, and better skilled workers have a comparative advantage in more complex jobs. The authors assume increasing returns to scale in the matching technology. As a result, workers' rate of contact does not depend on the stationary composition of skills.

${ }^{3}$ In Uren for instance, low-skilled workers may decide to seek complex jobs because their productivity is higher in such jobs, which reduces job creation in this sector and creates a crowding-out effect for the high-skilled. They massively decide to direct their search towards simple jobs. Decentralized matching is then non-assortative, while efficiency requires the allocation of complex jobs to the high-skilled workers.
} 
gains must be positive on each sub-market. In a model where education determines the scope of individual skills, Charlot et al (2005) also assume that the search market is segmented by technology, and workers only participate in market segments for which they have the relevant skills. However, individual productivity only depends on the technology, and there is no composition externality in their paper.

Finally, Albrecht et al (2006) consider a model of directed search with multiple applications and homogenous agents. They allow for the choice of an endogenous number of applications, and find that (whenever larger than 2) in equilibrium it is typically too large: workers search too much. While the result is similar to the one in this paper, the reason is entirely different. They highlight an externality on firms that process applications but end up losing the selected worker to a competitor. Both papers complement each other: job-seekers may well apply for too many jobs.

The paper is organized as follows. Section 2 presents the basic framework. In section 3 , I shed light on the composition externality implied by choosy search. Section 4 shows that workers prospect too many market segments. Section 5 formally compares the framework to MZ and SS. Section 6 concludes.

\section{The model}

The model closely follows MZ, except for the matching technology ${ }^{4}$. I consider the stationary state of a continuous time economy populated by a continuum of heterogenous firms and workers. Firms and workers differ with regard to their location on the space of technology. More specifically, workers are uniformly distributed on a circle with a perimeter of one. The total mass of workers is normalized to one. Heterogeneity in locations can be attributed to ex-ante differences in tastes or fields of knowledge. Each worker is either employed in a particular firm, or unemployed. There is no on-the-job search.

Firms are distributed along the same circle, yet their distribution is endogenous. I will show later that, like workers, they will be uniformly distributed ex-post in a symmetric equilibrium. Each firm has a unique position which can be either filled or vacant. Vacant positions incur a flow cost $c>0$ independent of the firm's location. Filled positions employ a worker and obtain a revenue from selling the output they produce. The price of the consumption good is normalized to one.

All agents are risk-neutral and $r$ is the rate of time preference, as well as the interest rate of the economy.

Production. The productivity of a pair depends negatively on the distance between the location of the worker and the location of the firm, what MZ term the mismatch technology. Formally,

$$
y_{i j} \equiv y(d(i, j))
$$

where $i(j)$ is the location of the worker (firm) and $d(i, j)$ is the absolute distance between $i$ and $j$ obtained by moving along the perimeter of the circle in the shortest direction. It satisfies $d(i, i)=0$ and $d(i, i+1 / 2)=1 / 2$. The mismatch technology is characterized by the following properties:

\footnotetext{
${ }^{4}$ There is also a minor difference concerning the production technology, which precludes the corner solution in which workers prospect jobs on the whole search market.
} 
Assumption 1 The function $y$ is strictly decreasing, with $y(0)=y_{0}>0$ and $y(1 / 2)=$ 0 .

The strict monotonicity of $y$ and $y(1 / 2)=0$ together will imply that there exists a critical distance above which firms and workers not only never match, but also never meet.

Matching sector. Here is the critical departure from MZ. MZ describe their model as a framework with a unique search place where all firms and workers meet. Alternatively, I suggest to formalize the search process as follows. The search market is segmented by location: job advertisements convey full information on the skill requirement, and there is a separate search sub-market for each location. The MZ model may then be interpreted as a framework where workers send their applications to all the different locations, regardless of whether they will accept an offer or not. By contrast, I assume that workers only apply on the sub-markets for which they are willing to take a position.

On each sub-market $j$, the flow of matches $M_{j}$ is given by the matching technology:

$$
M_{j} \equiv M\left(n_{j}, v_{j}\right)
$$

where $n_{j}$ and $v_{j}$ are, respectively, the number of active job-seekers and vacancies on market $j$. The function $M$ has the standard following properties:

Assumption 2 The function $M:[0,+\infty) \times[0,+\infty) \rightarrow[0,+\infty)$ (i) is strictly increasing in each of its arguments, strictly concave and admits constant returns to scale, (ii) satisfies the following boundary conditions: $M(z, 0)=M(0, z)=0$, for $z \geq 0$, and the Inada conditions.

Matches are equiprobably distributed among job-seekers, as well as among vacancies. Denoting by $\theta_{j} \equiv v_{j} / n_{j}$ the market-specific tightness, the flow probability that a worker receives an offer from market $j$ is $m\left(\theta_{j}\right) \equiv M\left(1, \theta_{j}\right){ }^{5}$ Symmetrically, the flow probability that a firm meets an applicant is $q\left(\theta_{j}\right)=m\left(\theta_{j}\right) / \theta_{j}$.

Firms' and workers' gains. Let $s$ be the exogenous job separation rate. In the remaining, workers and firms are identified by their locations $i$ and $j$ (resp.). Let $U_{i}$ and $W_{i j}$ be the asset values of an unemployed and an employed worker, respectively. Similarly, $J_{i j}$ and $V_{j}$ denote the values of a filled and of a vacant positions ${ }^{6}$. Finally, $w_{i j}$ is the wage paid to the worker. There is no unemployment benefit. Those values satisfy the standard

\footnotetext{
${ }^{5}$ More formally, the probability to receive an offer from the segment $[j, j+d j]$ is $m\left(\theta_{j}\right) d j d t$ over the time period $d t$. This makes clear that there cannot be multiple offers at a time: when $d j$ tends to 0 , the number of search places tends to infinity, but the contact rate specific to each place tends to 0 . In the Appendix, I demonstrate the impossibility of simultaneous offers in the case where the contact rate is the same in each location.

${ }^{6}$ As MZ, we keep the standard notation $J$ for the value of a filled position even though this may create confusion, as $j$ is the location of the firm.
} 
arbitrage equations:

$$
\begin{aligned}
r U_{i} & =\int_{i-1 / 2}^{i+1 / 2} \max \left\{m\left(\theta_{j}\right)\left[W_{i j}-U_{i}\right], 0\right\} d j \\
r W_{i j} & =w_{i j}+s\left[U_{i}-W_{i j}\right] \\
r J_{i j} & =y_{i j}-w_{i j}+s\left[V_{j}-J_{i j}\right] \\
r V_{j} & =-c+q\left(\theta_{j}\right) \mathbb{E}\left[\max \left\{J_{i j}-V_{j}, 0\right\}\right]
\end{aligned}
$$

Each contact generates a match surplus. When the match surplus is positive, it must be shared between the firm and the worker. I follow the standard approach in the literature and suppose that the wage is determined by Nash bargaining. This yields:

$$
\beta\left(J_{i j}-V_{j}\right)=(1-\beta)\left(W_{i j}-U_{i}\right)
$$

where $\beta \in(0,1)$ is the worker's exogenous bargaining power. This sharing rule implies that $W_{i j} \geq U_{i}$ if and only if $J_{i j} \geq V_{j}$.

The number of vacancies advertised on each search sub-market is determined by free entry. This implies all rents are null and drives the value of a vacancy down to zero, that is $V_{j}=0$ for all $j \in[0,1]$. This yields

$$
c / q\left(\theta_{j}\right)=\mathbb{E}\left[\max \left\{J_{i j}, 0\right\}\right]
$$

\section{Equilibrium}

In this section, I focus on the properties of the equilibrium. I only consider symmetric equilibria. I first describe the reservation wage property of the unemployed search strategy. Then, I compute tightness and solve for the general equilibrium.

Job-seekers' behaviour. Wages can be derived from equation (4), (5), (7):

$$
w_{i j}=\beta y_{i j}+(1-\beta) r U_{i}
$$

It follows the difference between the asset values of an employed and an unemployed worker satisfies

$$
(r+s)\left(W_{i j}-U_{i}\right)=\beta\left(y_{i j}-r U_{i}\right)
$$

Lemma 1 Let $\left\{\theta_{j}\right\}_{j=0}^{1}>0$ be given. For all $i$, there exists a unique $\sigma_{i} \in(0,1 / 2)$ such that

$$
\begin{gathered}
W_{i j} \geq U_{i} \text { iff } d(i, j) \leq \sigma_{i} \\
y\left(d\left(i, i+\sigma_{i}\right)\right)=r U_{i} \\
r U_{i}=\beta \int_{i-\sigma_{i}}^{i+\sigma_{i}} m\left(\theta_{j}\right) \frac{y_{i j}-r U_{i}}{r+s} d j
\end{gathered}
$$


Lemma 1 states the search behaviour follows a reservation wage-type of strategy. The surplus derived from a match is strictly decreasing in the distance between the location of the worker and that of the firm. The worker participates to all sub-markets yielding a positive match surplus. This defines a critical distance $\sigma_{i}$ below (above) which the worker (does not) participates. The critical distance equalizes the output flow derived from the limit location to the return to search.

As far as I do not impose anything but positivity to the sequence $\left\{\theta_{j}\right\}_{j=0}^{1}$, workers of different locations may have different reservation wage strategies, that is different critical distances $\sigma_{i}$. Taking account of these differences singularly complicates the analysis below. For this reason, I only focus on symmetric equilibria and suppose $\theta_{j}=\theta$ for all $j$. This implies that firms are uniformly distributed along the circle. Indeed, $\theta_{j}=\theta$ for all $j$ implies that $\sigma_{i}=\sigma$ for all $i$. Given that workers are uniformly distributed along the circle, the unnormalized density of job-seekers in each location is $n=2 u \sigma$, that is the number of unemployed times the search span in both directions. It follows that the unnormalized density of firms in each location is $v=\theta 2 u \sigma$, i.e. firms are uniformly distributed.

Despite they are heterogenous, all workers face the same market conditions. As a consequence, the return to search is the same for all, and so is the reservation strategy. The return to search $r U$ and critical distance $\sigma$ solve:

$$
\begin{aligned}
r U & =\frac{\beta m(\theta)}{r+s+2 \beta m(\theta) \sigma} \int_{-\sigma}^{\sigma} y(d(0, j)) d j \\
r U & =y(d(0, \sigma))
\end{aligned}
$$

Matching frictions make workers less selective. The critical distance $\sigma$ is thus decreasing in market tightness $\theta$.

From now on, I drop location-specific indices and summarize heterogeneity as follows: $U_{i}=U, W_{i j}=W(x), J_{i j}=J(x), y_{i j}=y(x), w_{i j}=w(x)$, where $x \equiv d(i, j)$.

Tightness determination. Assume $r U$ is given. From the wage equation (9), the value of a filled job with an $x$-worker is worth

$$
J(x)=(1-\beta) \frac{y(x)-r U}{r+s}
$$

Replacing (16) in the free entry equation (8) determines the tightness in each sector. I obtain:

$$
\frac{c}{q(\theta)}=(1-\beta) \frac{\bar{y}(\sigma)-r U}{r+s}
$$

where $\bar{y}(\sigma) \equiv \sigma^{-1} \int_{0}^{\sigma} y(x) d x$ is the average productivity among the job-seekers. Equation (17) defines common sector-specific tightness as an increasing function of the mean productivity of the job-seekers. As

$$
\bar{y}^{\prime}(\sigma)=\frac{y(\sigma)-\bar{y}(\sigma)}{\sigma}<0
$$

tightness is strictly decreasing in the critical distance $\sigma$. It follows that choosy search generates a composition externality, transiting through firms' willingness to post vacancies. When the return to search $r U$ rises, workers become more selective and $\sigma$ goes down. 
The average productivity of job seekers rises as a result. Due to rent-sharing, expected profits are increasing in average productivity, which encourages job creation.

Equilibrium. An equilibrium is composed of a return to search $r U$, a critical technological distance $\sigma$ and a tightness $\theta$ solving together (14), (15) and (17). Using the fact that $y(\sigma)=r U$, solving for an equilibrium reduces to find a pair $\left(\sigma^{*}, \theta^{*}\right)$ such that

$$
\begin{aligned}
y(\sigma) & =\frac{2 \beta m(\theta)}{r+s+2 \beta m(\theta) \sigma} \bar{y}(\sigma) \\
\frac{c}{q(\theta)} & =\frac{1-\beta}{r+s}[\bar{y}(\sigma)-y(\sigma)]
\end{aligned}
$$

(RS) is the reservation strategy curve stemming from workers' job search behaviour. It is downward sloping in the $(\sigma, \theta)$ plane, as workers get more choosy when tightness increases. (FE) is the free entry curve. Its slope is ambiguous, reflecting the two opposite effects of $\sigma$ on firms' profitability. On the one hand, an increase in $\sigma$ means a decrease in the return to search. In turn, outside options are lower at the wage bargaining stage, which improves firms' gains. This effect also happens in MZ. On the other hand, an increase in $\sigma$ deteriorates the sector-specific mean productivity of the job seekers, which reduces firms' profitability. This effect does not happen in MZ.

Proposition 1 (i) There exists a symmetric equilibrium

(ii) There exists a unique symmetric equilibrium if $y$ is concave

(iii) When the symmetric equilibrium is unique,

$$
d \sigma^{*} / d \beta \lesseqgtr 0 \text { iff } \beta-1+\alpha\left(\theta^{*}\right) \lesseqgtr 0
$$

where $\alpha(\theta) \equiv \theta m^{\prime}(\theta) / m(\theta) \in(0,1)$.

Existence follows from the continuity and the boundary properties of (RS) and (FE). As (FE) may have decreasing parts, uniqueness is not guaranteed. However, the equilibrium is unique when $y$ is concave in $x .^{7}$ Hence, the slope of $(\mathrm{FE})$ is always positive and there is a unique equilibrium; it is depicted by Figure 1.

\section{[Figure 1: Uniqueness of equilibrium]}

Property (iii) depicts the effect of the price of skills on workers' choosiness. Like the standard case with random search and job rejections, the impact of the bargaining power depends on the difference between the (exogenous) share of total match surplus obtained by the workers and their contribution to the matching technology, that is on $\beta-1+$ $\alpha\left(\theta^{*}\right)$. As $\alpha$ generally depends on tightness, and equilibrium tightness is parametrized by the bargaining power, this condition may be non linear, implying several local extrema. However, in the smooth case of a Cobb-Douglas matching technology, $\sigma^{*}$ is $\cup$-shaped in $\beta$, reaching its minimum in $\beta=1-\alpha$. This implies the extent of the mismatch is minimized when the so-called Hosios condition holds. This result is similar to the stochastic-job matching model with random search (see MZ and Pissarides, 2000).

\footnotetext{
${ }^{7}$ This case includes the linear mismatch technology chosen by MZ to illustrate their results.
} 


\section{Decentralized vs efficient allocation of talents}

In this section, I show that the decentralized allocation is never constrained efficient. Agents are always insufficiently selective, irrespective of bargaining power. As a result, they prospect a job on too many market segments.

As is standard, I consider the case where $r \rightarrow 0$. The social planner aims to maximize steady-state consumption, i.e. total output net of search costs (see Hosios, 1990, and Pissarides, 2000):

$$
\left(\theta^{p}, \sigma^{p}\right) \in \arg \max _{\theta \geq 0, \sigma \in[0,1 / 2]}\{(1-u) \bar{y}(\sigma)-c v\}
$$

where $\bar{y}(\sigma) \equiv \int_{0}^{\sigma} y(x) d x / \sigma$. The planner controls for sector-specific tightness $\theta$, and for the critical distance $\sigma$. The maximization program is subject to the Beveridge curve $u=s /(s+2 m(\theta) \sigma)$ and to the definition of tightness, which implies $v=2 \theta u \sigma$.

The first-order condition with respect to tightness is:

$$
-\frac{\partial u}{\partial \theta} \bar{y}-c \frac{\partial v}{\partial \theta}=0
$$

While choosing the number of vacancies, the planner balances the two standard search externalities arising from constant returns to scale in the matching technology: advertising an additional vacancy translates into a rise in workers' job-finding rate, but into a fall in firms' recruitment rate.

The first-order condition with respect to critical distance is:

$$
-\frac{\partial u}{\partial \sigma} \bar{y}+(1-u) \frac{\partial \bar{y}}{\partial \sigma}-c \frac{\partial v}{\partial \sigma}=0
$$

As usual, the first term is the gain following the decrease in the unemployment rate, and the second term is the loss in average productivity. The novelty comes from the third term, which takes into account the impact of $\sigma$ on search costs. That is,

$$
\frac{\partial v}{\partial \sigma}=2 \theta \sigma \frac{\partial u}{\partial \sigma}+2 \theta u=2 \theta u^{2}>0
$$

The critical distance raises congestion as each worker participates to a larger number of markets.

Manipulating the different equations, I finally get the following characterization of the efficient allocation:

$$
\begin{gathered}
\frac{y\left(\sigma^{p}\right)}{s}=\frac{\left(1-\alpha^{p}\right) 2 m\left(\theta^{p}\right) \sigma^{p}}{s+\left(1-\alpha^{p}\right) 2 m\left(\theta^{p}\right) \sigma^{p}} \frac{\bar{y}\left(\sigma^{p}\right)}{s}+c / q\left(\theta^{p}\right) \\
c / q\left(\theta^{p}\right)=\frac{\alpha^{p} \bar{y}\left(\sigma^{p}\right)}{s+\left(1-\alpha^{p}\right) 2 m\left(\theta^{p}\right) \sigma^{p}}
\end{gathered}
$$

where $\alpha^{p} \equiv \theta^{p} m^{\prime}\left(\theta^{p}\right) / m\left(\theta^{p}\right)$. Equations (23) and (24) must be compared to equations $(\mathrm{RS})$ and $(\mathrm{FE})$, which characterize the decentralized allocation. The right-hand side of equation (23) is split into two terms to ease the comparison.

Proposition $2 \sigma^{*}>\sigma^{p}$ for all $\beta \in[0,1]$ 
Unlike MZ, the decentralized allocation cannot replicate the efficient allocation, even when the Hosios condition holds. There is a simple way to see it. We know from Proposition 1 that $\sigma^{*}$ is minimized when the Hosios condition holds. In that case, the decentralized allocation and the efficient allocation obey very similar equations, but the second term on the right-hand side of equation (23). Indeed, the planner chooses $\sigma^{p}$ so that the discounted output flow of the least productive worker in a particular location covers the opportunity cost of working in the corresponding sector, plus the average search spending in that search place. This second term implies that $y\left(\sigma^{p}\right)>y\left(\sigma^{*}\right)$, which is equivalent to $\sigma^{p}>\sigma^{*}$. Indeed, there is an additional externality to the standard stochastic-job matching model. Increasing $\sigma$ raises congestion effects in each market, and deteriorates the quality of job-seekers in each sector through a composition effect. The planner takes this effect into account, and therefore sets a lower $\sigma$.

The question remains to know why firms hire workers whose productivity is insufficient to cover the average search cost incurred to recruit them. The reason relies on the fact that search costs are sunk at the time of hiring the worker. Hence, matches that are ex-ante inefficient become profitable ex-post. If firms were able to commit on a minimum skill level, workers would not search jobs in locations that are too far from their own and the Hosios condition would decentralize the efficient allocation.

To distinguish the distortions induced by choosy search from the distortions due to inadequate bargaining power, I now address the following question: at given $\beta, r \rightarrow 0$, what is the efficient choice of $\sigma$ ? The planner still maximizes steady-state consumption. Its unique instrument is the critical technological distance $\sigma$, while tightness results from firms' choice of entry on the search market. Formally, the maximization program is the following:

$$
\widetilde{\sigma} \in \arg \max _{\sigma \in[0,1 / 2]}\{(1-u) \bar{y}(\sigma)-c v\}
$$

subject to the definition of tightness $\theta=v /(2 u \sigma)$, the Beveridge curve $u=s /(s+2 m(\theta) \sigma)$, and the free entry condition $c / q(\theta)=(1-\beta)[\bar{y}(\sigma)-r U] / s$, where $r U$ stands for $\lim _{r \rightarrow 0} r U$. The problem (25) is equivalent to ${ }^{8}$

$$
\widetilde{\sigma} \in \arg \max _{\sigma \in[0,1 / 2]} r U
$$

subject to $c / q(\theta)=(1-\beta)[\bar{y}(\sigma)-r U] / s$. The objective is simply the return to search of a representative job-seeker ${ }^{9}$. However, and unlike individuals, the planner takes into account the fact that changes in $\sigma$ alter the composition of job seekers in each sub-market, and therefore affect tightness. The first-order condition can be written as follows:

$$
y(\widetilde{\sigma})=r U+\frac{\alpha(\theta)}{1-\alpha(\theta)}[\bar{y}(\widetilde{\sigma})-y(\widetilde{\sigma})]>r U
$$

where $\alpha(\theta) \equiv \theta m^{\prime}(\theta) / m(\theta)$. The social return from the critical technological distance is lower than the private return, and the planner sets a lower $\sigma$ than the one chosen by individuals. Importantly, such a result cannot arise in MZ, where job rejection is actually efficient at given bargaining power.

\footnotetext{
${ }^{8}$ Here, it is implicit that $r U$ stands for the return to search at given $\sigma$, a (small) departure from (3).

${ }^{9}$ This is a general property in matching models. See Pissarides (1986).
} 


\section{Understanding the results}

In this section, I slightly alter my model to compare it to MZ and SS. In the former case, I introduce some imperfection in workers' ability to sort vacancies by job type. In the latter case, I introduce sector-specific search intensity.

Comparing with MZ. In MZ, search is random, and workers can meet firms from any location on the technological circle. Individuals have ex-ante differences, but they do not act on the knowledge of comparative advantages. They wait to match, and then decide. The model is formally equivalent to Pissarides (1985) stochastic job matching model with ex-post heterogeneity (see Pissarides, 2000). There are no composition externality, and the Hosios condition decentralizes the efficient allocation both in Pissarides and MZ.

I introduce some imperfection in the filter agents use to sort the vacancies. With probability $\varepsilon \in[0,1]$, the filter does not work and the unemployed gets an offer from an arbitrary location on the circle - a random offer. With the remaining probability $1-\varepsilon$, the filter returns a job offer from the pool of prospected markets - a choosy offer. Choosy search means $\varepsilon=0$, while $\varepsilon=1$ corresponds to MZ.

An unemployed must take two decisions: Which set of markets to prospect, and whether to accept a potential random offer or not. Let $\sigma_{c s}$ denote the critical distance corresponding to the farthest prospected market. Similarly, $\sigma_{m z}$ is the farthest accepted job offer. The value of unemployment and the value of a vacancy are now expressed as:

$$
\begin{aligned}
r U= & \max _{\sigma_{c s}, \sigma_{m z}} \frac{2 \beta m(\theta)}{r+s}\left\{(1-\varepsilon) \sigma_{c s}\left[\bar{y}\left(\sigma_{c s}\right)-r U\right]+\varepsilon \sigma_{m z}\left[\bar{y}\left(\sigma_{m z}\right)-r U\right]\right\} \\
r V= & -c+q(\theta)\{(1-\varepsilon) \mathbb{E}[J(x) \mid J(x) \geq V] \\
& +\varepsilon \operatorname{Pr}(J(x) \geq V) \mathbb{E}[J(x) \mid J(x) \geq V]-V\}
\end{aligned}
$$

With probability $1-\varepsilon$, the firm receives a choosy application. Such application always leads to an employment relationship, as the worker makes sure ex-ante that each meeting is mutually acceptable. With probability $\varepsilon$, the firm receives a random application. This type of applications leads to an employment relationship if and only if the distance separating the worker and the firm is smaller than the critical distance $\sigma_{m z}$. Such an event happens with probability $\operatorname{Pr}(J(x) \geq V)=\operatorname{Pr}\left(x \leq \sigma_{m z}\right)=\sigma_{m z}$. This term is the key difference between MZ and the choosy search model. Other asset equations remain unchanged.

A representative unemployed chooses $\sigma_{c s}$ and $\sigma_{m z}$ so that $y\left(\sigma_{c s}\right)=y\left(\sigma_{m z}\right)=r U$. The worker sets the same critical distance $\sigma$, resulting in each case from a no-surplus condition. Noteworthy, the private choice of $\sigma_{m z}$ does not involve any externality, while $\sigma_{c s}$ still generates the composition externality identified in the previous section.

To show this, I use the free entry condition $V=0$ to get

$$
\frac{c}{q(\theta)}=\frac{1-\beta}{r+s}\left\{(1-\varepsilon)\left[\bar{y}\left(\sigma_{c s}\right)-r U\right]+\varepsilon \sigma_{m z}\left[\bar{y}\left(\sigma_{m z}\right)-r U\right]\right\}
$$


This equation defines tightness as a function $\theta=\theta\left(\sigma_{c s}, \sigma_{m z}\right)$, with

$$
\begin{gathered}
\frac{\partial \theta\left(\sigma_{c s}, \sigma_{m z}\right)}{\partial \sigma_{c s}} \stackrel{\text { sign }}{=} \frac{y\left(\sigma_{c s}\right)-\bar{y}\left(\sigma_{c s}\right)}{\sigma_{c s}}<0 \\
\frac{\partial \theta\left(\sigma_{c s}, \sigma_{m z}\right)}{\partial \sigma_{m z}} \stackrel{\text { sign }}{=} y\left(\sigma_{m z}\right)-r U
\end{gathered}
$$

Using $y\left(\sigma_{c s}\right)=y\left(\sigma_{m z}\right)=r U$, the latter equation becomes $\partial \theta(\sigma, \sigma) / \partial \sigma_{m z}=0 . \mathrm{A}$ marginal decrease in $\sigma_{c s}$ raises tightness, while a marginal decrease in $\sigma_{m z}$ does not affect it. Intuitively, $\sigma_{m z}$ maximizes the total surplus accruing from a contact. Given that the value of a vacancy positively depends on such surplus, $\sigma_{m z}$ maximizes both the value of unemployment and the value of a vacancy. Put differently, the choosy search behaviour is inefficient because it generates composition effects, while the random search behaviour is efficient because it does not alter the distribution of job seekers in each market.

To confirm this intuition, I consider the planner's choices, taking as given the bargaining power and the tightness resulting from free entry. I focus on the case where $r \rightarrow 0$. Efficient choices result from

$$
r U=\max _{\sigma_{c s}, \sigma_{m z}} \frac{2 \beta m\left(\theta\left(\sigma_{c s}, \sigma_{m z}\right)\right)}{s}\left\{(1-\varepsilon) \sigma_{c s}\left[\bar{y}\left(\sigma_{c s}\right)-r U\right]+\varepsilon \sigma_{m z}\left[\bar{y}\left(\sigma_{m z}\right)-r U\right]\right\}
$$

This yields the following first-order conditions:

$$
\begin{aligned}
y\left(\widetilde{\sigma}_{c s}\right) & =r U+\frac{\alpha(\theta)}{1-\alpha(\theta)}\left[\bar{y}\left(\widetilde{\sigma}_{c s}\right)-y\left(\widetilde{\sigma}_{c s}\right)\right]>r U \\
y\left(\widetilde{\sigma}_{m z}\right) & =r U
\end{aligned}
$$

Three properties can be derived from such conditions. First, the decentralized equilibrium is inefficient. Second, the planner sets two different critical distances. Like individuals, the planner chooses the threshold $\sigma_{m z}$ so as to equalize the worst output to the return to search. However, the planner sets a lower threshold $\sigma_{c s}$ to account for its negative impact on job creation. Finally, note that both $\sigma_{c s}$ and $\sigma_{m z}$ are too high compared to the planner's solution. Indeed, $\sigma_{m z}$ does not respond to the correct incentives as $r U$ is inefficiently low. The magnitude of such inefficiency depends on $\varepsilon$. When $\varepsilon$ is close to 1 , the worker mainly receives random offers, so that the return to search is only marginally affected by the critical distance $\sigma_{c s}$.

Comparing with SS. In SS, agents also have ex-ante differences, but they differ in skill level. To simplify, they are good, or bad. There is a single search market, and individuals choose their search intensity. SS show that bad agents search too intensively, while good agents undertake too low search investments ${ }^{10}$. My model can replicate this result on each market of the continuum $[0,1]$. I let the agents choose their search intensity on each sub-market ${ }^{11}$. The composition externality then implies that the unemployed search too much in sectors far from their technological location, while they search too little in closer

\footnotetext{
${ }^{10} \mathrm{SS}$ also show that high-skilled (low-skilled) workers reject job offers too rarely (frequently).

${ }^{11}$ In a partial equilibrium model of regional segmentation, Burda and Profit (1996) consider a similar exercise in which search costs vary with the distance between worker's location and the prospected search market.
} 
sectors. Put differently, workers do not search enough where they have a comparative advantage - where they are good -, and search too much where they do not - where they are bad.

Let $\lambda(x)$ denote search intensity on sub-market (located in) $x$. I assume the search cost is linear in $\lambda(x)$, while the search technology has decreasing marginal returns. The search intensity $\lambda \operatorname{costs} \kappa \lambda$, with $\kappa>0$, while it offers $f(\lambda)$ efficiency units of search, with $f(0)=f^{\prime}(0)=0, f^{\prime}(\lambda)>0$, and $f^{\prime \prime}(\lambda)<0$ for all $\lambda>0$.

The individual pattern of search intensity by sector is derived from the following maximization program:

$$
r U=\max _{\{\lambda(.)\}}\left\langle 2 \int_{0}^{1 / 2}[m(\theta) f(\lambda(x))(W(x)-U)-\kappa \lambda(x)] d x\right\rangle
$$

Other asset equations remain unchanged. The first-order conditions together with the wage equation yield:

$$
\beta m(\theta) f^{\prime}(\lambda(x)) \frac{y(x)-r U}{r+s}=\kappa \text { for all } x \leq \sigma
$$

where $y(\sigma)=r U$.

Proposition 3 The optimal search intensity $\lambda(x,$.$) is such that$

(i) $\lambda_{x}(x,)<$.0 for all $x \in[0, \sigma]$

(ii) There exists a unique $\widehat{x} \in[0, \sigma]$ such that $\lambda_{\theta}(x,.) \gtreqless 0$ iff $x \lesseqgtr \widehat{x}$

The decrease in search intensity with technological distance simply reflects the decrease in wage due to lower productivity. The main implication is that for a particular sector, there is a continuous distribution of types, and search intensity is strictly increasing in worker's type/sector-specific productivity. This result mimics SS, where the good search more than bad. Moreover, search intensity is negatively related to tightness in sectors where occupations are ill-suited to the worker, and positively related to tightness in sectors where occupations fit better the worker's skills. Indeed, tightness exerts two conflicting effects on search intensity. The first effect is sector-specific: tightness directly raises the marginal benefit to search effort, which tends to increase search intensity. The second effect is indirect: tightness raises the value of unemployment, which tends to reduce search efforts. The former effect dominates the latter at low values of $x$, and is dominated at higher values. This property is a consequence of symmetric comparative advantages. It differs from SS, in which tightness increases search investments irrespective of the skill levels. In their framework, the indirect effect is always dominated by the direct effect, because workers having a lower productivity also have a lower value of unemployment.

To close the model, I use the free entry condition to get

$$
\frac{c}{q(\theta)}=\frac{1-\beta}{r+s}[\bar{y}-r U]
$$

where $\bar{y} \equiv \int_{0}^{\sigma} f(\lambda(x)) y(x) d x\left[\int_{0}^{\sigma} f(\lambda(x)) d x\right]^{-1}$ is the mean productivity among the job seekers of a given sector. This is a functional that depends on the whole path of sectorspecific search intensities $\{\lambda()$.$\} . Equation (33) shows the interest of this reformulation$ 
of SS. In SS, different workers have different search efforts and reservation wages. As a result, one must account for the endogenous distribution of workers' skills. By contrast, workers share the same reservation wage $r U$ in my model, which simplifies the analysis.

Taking the Volterra derivative of the functional $\bar{y}$ with respect to a small variation of $\lambda(z)$ in the neighbourhood of the distance $x$ yields:

$$
\bar{y}^{\prime}(\lambda(z), x)=\frac{f^{\prime}(\lambda(x))}{\int_{0}^{\sigma} f(\lambda(z)) d z}[y(x)-\bar{y}]
$$

It follows that tightness is increasing (decreasing) in the search intensity of workers whose productivity is above (below) the average. Together with property (ii) of proposition 3 , this latter result offers a new multiplier effect. By searching more intensively in sectors that best fit their characteristics, workers improve tightness. This in turn makes them search less intensively in distant sectors, and more intensively in closer sectors.

To analyze the efficiency properties of the decentralized equilibrium, I study the search intensities that would be chosen by a social planner, taking as given workers' bargaining power and accounting for changes in tightness resulting from firms' free entry on the matching market. I consider the case where $r \rightarrow 0$. The socially efficient search intensities result from

$$
\max _{\lambda(.)}\{r U\}
$$

subject to $c / q(\theta)=(1-\beta)(\bar{y}(\{\lambda()\})-.r U) / s$. The first-order conditions to the maximization program yield

$$
\frac{\beta m(\theta) f^{\prime}(\lambda(x))}{s}\left\{y(x)-r U+\frac{\alpha(\theta)}{1-\alpha(\theta)}(y(x)-\bar{y})\right\}=\kappa
$$

The social marginal return to search is higher than the private marginal return when $y(x)>\bar{y}$, that is when the worker improves the mean productivity among the job seekers. Conversely, it is lower than the private marginal return when $y(x)<\bar{y}$. It follows that workers search too little on market segments where they have a comparative advantage, and too much on market segments where they have a comparative disadvantage. This result parallels SS, where the good agents search too little and the bad agents search too much.

\section{Conclusion}

This paper proposes a multi-sector matching model with ex-post Nash bargaining where workers have (symmetric) sector-specific skills à la Salop (1979). The search market is segmented by sector and workers choose whether or not to participate to each sector. In this framework, searching for a job involves deciding how far to search. I show that such a search behaviour generates a composition externality: while choosing the range of markets where they participate, workers do not internalize the impact of their choice on the mean skill level among the workforce attached to each sector. As a result workers search too much, i.e. in too many sectors, and the decentralized allocation of talents cannot replicate the efficient allocation, irrespective of the bargaining power. 
The model may be extended along several directions. First, one may introduce a minimum wage. Intuitively, the minimum wage may help to improve the allocation of workers to jobs by making low productivity pairs non-profitable. Secondly, one may endogenize the distribution of skills across sectors, through, say, schooling. The likely result of such an analysis is that workers may well invest the right amount in schooling, but allocate it wrongly between the different sectors. Those extensions are on my research agenda. 


\section{APPENDIX}

Proof of lemma 1 Replacing equation (10) into equation (3) I get:

$$
r U_{i}=\int_{i-1 / 2}^{i+1 / 2} \max \left\{\frac{\beta m\left(\theta_{j}\right)}{r+s}\left(y_{i j}-r U_{i}\right), 0\right\} d j
$$

Lemma 1 follows from Assumption 1, namely from the facts that $y_{i j} \equiv y(d(i, j))$ is continuous and strictly decreasing in $d(i, j)$ from $y_{0}$ to 0 .

Proof of proposition 1 An equilibrium is a non-negative vector $\left(\sigma^{*}, \theta^{*}\right)$ such that

$$
\begin{aligned}
m(\theta) & =\frac{r+s}{2 \beta} \frac{y(\sigma)}{\sigma[\bar{y}(\sigma)-y(\sigma)]} \\
\frac{c}{q(\theta)} & =\frac{1-\beta}{r+s}[\bar{y}(\sigma)-y(\sigma)]
\end{aligned}
$$

To show (i), I simply prove equations (38) and (39) define two loci in the plane $(\sigma, \theta)$ that intersect at least once.

Step 1. Equation (38) defines an implicit function $\theta_{1} \equiv \Theta_{1}(\sigma)$; it is continuous on $(0,1 / 2]$, strictly decreasing, and such that $\lim _{\sigma \rightarrow 0} \Theta_{1}(\sigma)=+\infty$ and $\Theta_{1}(1 / 2)=0$.

The existence, uniqueness and continuity of the function $\Theta_{1}$ over the range $(0,1 / 2]$ result from Assumption 2, which implies that $m$ is strictly increasing from 0 to infinity. In addition, $d \Theta_{1}(\sigma) / d \sigma$ has the sign of

$$
y^{\prime}(\sigma) \sigma[\bar{y}(\sigma)-y(\sigma)]+y(\sigma) \sigma y^{\prime}(\sigma)=\sigma y^{\prime}(\sigma) \bar{y}(\sigma)<0
$$

The fact that $\Theta_{1}(1 / 2)=0$ is induced by Assumption 2, which implies that $m(0)=$ 0 . Finally, $y(0)=y_{0}>0$, while L'Hôpital's rule implies that

$$
\lim _{\sigma \rightarrow 0} \sigma[\bar{y}(\sigma)-y(\sigma)]=0
$$

The fact that $\lim _{\sigma \rightarrow 0} \Theta_{1}(\sigma)=+\infty$ results from Assumption 2, which also implies that $\lim _{\theta \rightarrow+\infty} m(\theta)=+\infty$.

Step 2. Equation (39) defines an implicit function $\theta_{2} \equiv \Theta_{2}(\sigma)$; it is continuous on $[0,1 / 2]$, and such that $\Theta_{2}(0)=0$ and $\Theta_{2}(1 / 2)>0$.

The existence and uniqueness of the function $\Theta_{2}$ follow from Assumption 2. Using L'Hôpital's rule,

$$
\lim _{\sigma \rightarrow 0} \bar{y}(\sigma)=y_{0} \text { and } \lim _{\theta \rightarrow 0} 1 / q(\theta)=\lim _{\theta \rightarrow 0} 1 / m^{\prime}(\theta)=0
$$

Therefore, $\Theta_{2}(0)=0$. Moreover, $\bar{y}(1 / 2)>y(1 / 2)=0$. It follows that $\Theta_{2}(1 / 2)>0$. Step 3. Conclusion. 
An equilibrium solves $\theta^{*}=\Theta_{1}\left(\sigma^{*}\right)=\Theta_{2}\left(\sigma^{*}\right)$. The existence of equilibrium follows from step 1 and step 2. Namely, the continuity and the limit values of functions $\Theta_{1}$ and $\Theta_{2}$ imply that there exist $\sigma^{*} \in(0,1 / 2)$ such that $\Theta_{1}\left(\sigma^{*}\right)=\Theta_{2}\left(\sigma^{*}\right)$.

(ii) To prove claim (ii), it is sufficient to show that the curve (FE) is upward sloping for all $\sigma \in[0,1 / 2]$. Let $z(\sigma)=\bar{y}(\sigma)-y(\sigma)$; $\Theta_{2}^{\prime}(\sigma)$ has the sign of $z^{\prime}(\sigma)$. But

$$
z^{\prime}(\sigma)=-\frac{z(\sigma)}{\sigma}-y^{\prime}(\sigma)
$$

Suppose that (ii) is not true. Hence, there exists some $s \in(0,1)$ such that $z^{\prime}(s)<0$. However,

$$
z^{\prime \prime}(\sigma)=\frac{z(\sigma)-\sigma z^{\prime}(\sigma)}{\sigma^{2}}-y^{\prime \prime}(\sigma)
$$

Since $y$ is concave and $z^{\prime}(s)<0, z^{\prime \prime}(s)>0$. By induction, $z^{\prime}(\sigma)<0$ for all $\sigma \leq s$. In particular, $z^{\prime}(0)<0$, which is impossible because $z(0)=0$ and $z(\sigma)>0$ for all $\sigma>0$.

(iii) To sign the effect of $\beta$, it is necessary to differentiate the system (RS)-(FE). From step 1 of the proof of claim (i), we have

$$
\frac{c}{q\left(\Theta_{1}(\sigma, \beta)\right)}=\frac{1-\beta}{r+s}[\bar{y}(\sigma)-y(\sigma)]
$$

where the dependence of function $\Theta_{1}$ vis-à-vis $\beta$ has been made explicit. As the equilibrium is unique by assumption, $d \sigma^{*} / d \beta$ has the sign of

$$
A \equiv-\frac{c}{q^{2}} q^{\prime} \frac{\partial \Theta_{1}}{\partial \beta}+\frac{\bar{y}(\sigma)-y(\sigma)}{r+s}
$$

Using the fact that $\theta m^{\prime}(\theta) / m(\theta)=\alpha(\theta)$,

$$
\frac{\partial \Theta_{1}}{\partial \beta}=-\frac{\theta}{\alpha(\theta) \beta}
$$

Using the fact that $-\theta q^{\prime}(\theta) / q(\theta)=1-\alpha(\theta)$, I get

$$
A=\frac{1}{\alpha\left(\theta^{*}\right) \beta} \frac{\bar{y}(\sigma)-y(\sigma)}{r+s}\left[\beta-\left(1-\alpha\left(\theta^{*}\right)\right)\right]
$$

which shows (iii).

Proof of proposition 2 I first write the return to search as a function of bargaining power, tightness, and critical distance $\sigma$, i.e. $r U(\beta, \theta, \sigma)$ so that

$$
r U(\beta, \theta, \sigma)=2 \beta m(\theta) \frac{\bar{y}(\sigma)-r U(\beta, \theta, \sigma)}{s}
$$

The efficient allocation is such that

$$
\left(\theta^{p}, \sigma^{p}\right)=\arg \max _{\theta \geq 0, \sigma \in[0,1 / 2]}\left\{(1-u) \bar{y}(\sigma)-\frac{c}{q(\theta)} 2 m(\theta) u \sigma\right\}
$$


subject to $u=s /(s+2 m(\theta) \sigma)$. The first-order condition with respect to tightness yields:

$$
\frac{c}{q(\theta)}=\frac{\alpha(\theta) \bar{y}(\sigma)}{s+(1-\alpha(\theta)) 2 m(\theta) \sigma}
$$

Let $\theta_{p}(\sigma)$ be the implicit function solving the latter condition. The maximisation problem becomes

$$
\sigma^{p}=\arg \max _{\sigma \in[0,1 / 2]}\left\{r U\left(1-\alpha\left(\theta_{p}(\sigma)\right), \theta_{p}(\sigma), \sigma\right)\right\}
$$

with $\theta^{p}=\theta_{p}\left(\sigma^{p}\right)$.

Now, let $\theta_{*}(\beta, \sigma)$ denote the implicit function solving

$$
\frac{c}{q(\theta)}=\frac{1-\beta}{s}[\bar{y}(\sigma)-r U(\beta, \theta, \sigma)]
$$

The decentralized allocation is such that:

$$
y\left(\sigma^{*}\right)=r U\left(\beta, \theta_{*}\left(\beta, \sigma^{*}\right), \sigma^{*}\right)
$$

Now, let $(\widehat{\beta}, \widehat{\sigma}) \in \arg \max _{\beta \in[0,1], \sigma \in[0,1 / 2]} r U\left(\beta, \theta_{*}(\beta, \sigma), \sigma\right)$. Using the first-order condition and the definition (**) of $\sigma^{p}$ and $\theta^{p}$, I obtain $\widehat{\beta}=1-\alpha\left(\theta^{p}\right)$, and $\widehat{\sigma}=\sigma^{p}$. In addition,

$$
\begin{aligned}
y\left(\sigma^{p}\right) & =r U\left(1-\alpha^{p}, \theta_{*}\left(1-\alpha^{p}, \sigma^{p}\right), \sigma^{p}\right)+\frac{\alpha^{p} s}{s+\left(1-\alpha^{p}\right) 2 m\left(\theta^{p}\right) \sigma^{p}} \bar{y}\left(\sigma^{p}\right) \\
& >r U\left(1-\alpha^{p}, \theta_{*}\left(1-\alpha^{p}, \sigma^{p}\right), \sigma^{p}\right) \geq r U\left(\beta, \theta_{*}\left(\beta, \sigma^{*}\right), \sigma^{*}\right)=y\left(\sigma^{*}\right)
\end{aligned}
$$

with $\alpha^{p}=\alpha\left(\theta^{p}\right)$ and $\beta \in[0,1]$. It follows that $\sigma^{p}<\sigma^{*}$.

Proof of proposition 3 (i) comes from the facts that $f^{\prime \prime}<0$ and $y^{\prime}<0$.

(ii) Note that

$$
\operatorname{sign}\left\{\frac{\partial \lambda(x, .)}{\partial m(\theta)}\right\}=\operatorname{sign}\left\{y(x)-y(\sigma)-m(\theta) \frac{\partial r U}{\partial m(\theta)}\right\}
$$

with

$$
\frac{\partial r U}{\partial m(\theta)}=\frac{\beta \int_{0}^{\sigma} f(\lambda(x)) d x}{r+s+\beta m(\theta) \int_{0}^{\sigma} f(\lambda(x)) d x}[\bar{y}-y(\sigma)]
$$

It comes

$$
\begin{aligned}
\operatorname{sign}\left\{\frac{\partial \lambda(x, .)}{\partial m(\theta)}\right\} & =\operatorname{sign}\left\{y(x)-y(\sigma)-\frac{\beta m(\theta) \int_{0}^{\sigma} f(\lambda(x)) d x}{r+s+\beta m(\theta) \int_{0}^{\sigma} f(\lambda(x)) d x}[\bar{y}-y(\sigma)]\right\} \\
& =\operatorname{sign}\{F(x)\}
\end{aligned}
$$

But $F_{x}<0, F(\sigma)<0$ and

$$
F(0)>\frac{r+s}{r+s+\beta m(\theta) \int_{0}^{\sigma} f(\lambda(x)) d x}\left[y_{0}-y(\sigma)\right]
$$

Claim (ii) follows. 
Proof that individuals cannot receive multiple offers at the same time In this Appendix, I prove that workers searching on a continuum of independent matching places cannot receive more than one offer at a time. I prove this in the particular case where the contact rate is the same in each place, which corresponds to the symmetric equilibrium studied in the paper. Without loss of generality, let $I=[0,1]$ and cut it into $n$ discrete intervals of equal measure $1 / n$. Assume that the probability to receive an offer from any interval is $\mu d t / n$ over the period $d t$. I claim that

(i) As $n$ tends to infinity, the probability to receive an offer over any $d t<<\infty$ is strictly lower than 1

(ii) As $d t$ tends to 0 , the probability to receive more than one offer at a time is 0

(iii) The result holds when $n$ tends to infinity

Proof of (i). Let $X$ be the number of offers received over the period $d t$. The probability to receive $k \in\{0, \ldots, n\}$ offers is:

$$
P(X=k)=C_{n}^{k}(\mu d t / n)^{k}(1-\mu d t / n)^{n-k}
$$

As $1 / n \rightarrow 0$, it tends to

$$
P(X=k)=e^{-\mu d t} \frac{(\mu d t)^{k}}{k !}
$$

Therefore,

$$
P(X=0)=e^{-\mu d t}>0
$$

Proof of (ii). We have

$$
\begin{aligned}
p & =P(X=1 \mid X \geq 1)=\frac{P(X=1)}{P(X \geq 1)} \\
& =\frac{\mu d t(1-\mu d t / n)^{n-1}}{1-(1-\mu d t / n)^{n}}
\end{aligned}
$$

As $d t \rightarrow 0$, we finally have

$$
p \rightarrow \frac{\mu d t[1-\mu(1-1 / n) d t]}{\mu d t}=1
$$

Proof of (iii). Note that the latter proof holds for all $n$ and remains true for $n \rightarrow \infty$. In addition,

$$
P(X=1) \rightarrow e^{-\mu d t} \mu d t
$$

and

$$
P(X \geq 1) \rightarrow 1-e^{-\mu d t}
$$

Thus,

$$
p \underset{d t \rightarrow 0}{\rightarrow} \frac{e^{-\mu d t}}{1-e^{-\mu d t}} \mu d t=1
$$




\section{References}

[1] Albrecht, J., Gautier, P., Vroman, S., 2006. Equilibrium directed search with multiple applications. Review of Economic Studies 73, 869-891

[2] Barlevy, G., 2002. The sullying effect of recessions. Review of Economic Studies 69, $65-96$

[3] Burda, M., Profit, S., 1996. Matching across space: evidence on mobility in Czech Republic. Labour Economics 3, 255-278

[4] Charlot, O., Decreuse, B., 2005. Self-selection in education with matching frictions. Labour Economics 12, 251-267

[5] Charlot, O., Decreuse, B., Granier, P., 2005. Adaptability, productivity and the educational incentives in a matching model. European Economic Review 49, 10071032

[6] Diamond, P., 1982. Wage determination and efficiency in search equilibrium. Review of Economic Studies 49, 217-227

[7] Gautier, P., Teulings, C.N., 2004. The right man for the job. Review of Economic Studies 71-2, 553-580

[8] Hosios, A., 1990. On the efficiency of matching and related models of search and unemployment. Review of Economic Studies 57, 279-298

[9] Kiyotaki, N., Wright, R., 1993. A search-theoretic approach to monetary Economics. American Economic Review 83, 63-77

[10] Lockwood, B., 1986. Transferable skills, job matching, and the inefficiency of the natural rate of unemployment. Economic Journal 96, 961-974

[11] Marimon, R., Zilibotti, F., 1999. Unemployment versus mismatch of talents: reconsidering unemployment benefits. Economic Journal 109, 266-291

[12] Moscarini, G., 2001. Excess worker reallocation. Review of Economic Studies 68, 593-612

[13] Pissarides, C., 1985. Short run equilibrium dynamics of unemployment, vacancies and real wages. American Economic Review 75, 676-690

[14] Pissarides, C., 1986. Trade unions and the inefficiency of the natural rate of unemployment. Journal of Labor Economics 4, 582-595

[15] Pissarides, C., 2000. Equilibrium unemployment theory. MIT Press

[16] Salop, S., 1979. Monopolistic competition with outside goods. Bell Journal of Economics 10, 141-156

[17] Shi, S., 1997. Money and specialization. Economic Theory 10, 99-133 
[18] Shi, S., 2001. Frictional assignment. I. Efficiency. Journal of Economic Theory 98, $232-260$

[19] Shimer, R., 2005. The assignment of workers to jobs in an economy with coordination frictions. Journal of Political Economy 113, 996-1025

[20] Shimer, R., Smith, L., 2001. Matching, search and heterogeneity. Advances in Macroeconomics (B.E. Journal in Macroeconomics) 1, article 5

[21] Uren, L., 2006. The allocation of labour and endogenous search decisions. Topics in Macroeconomics (B.E. Journal in Macroeconomics) 6, article 13 


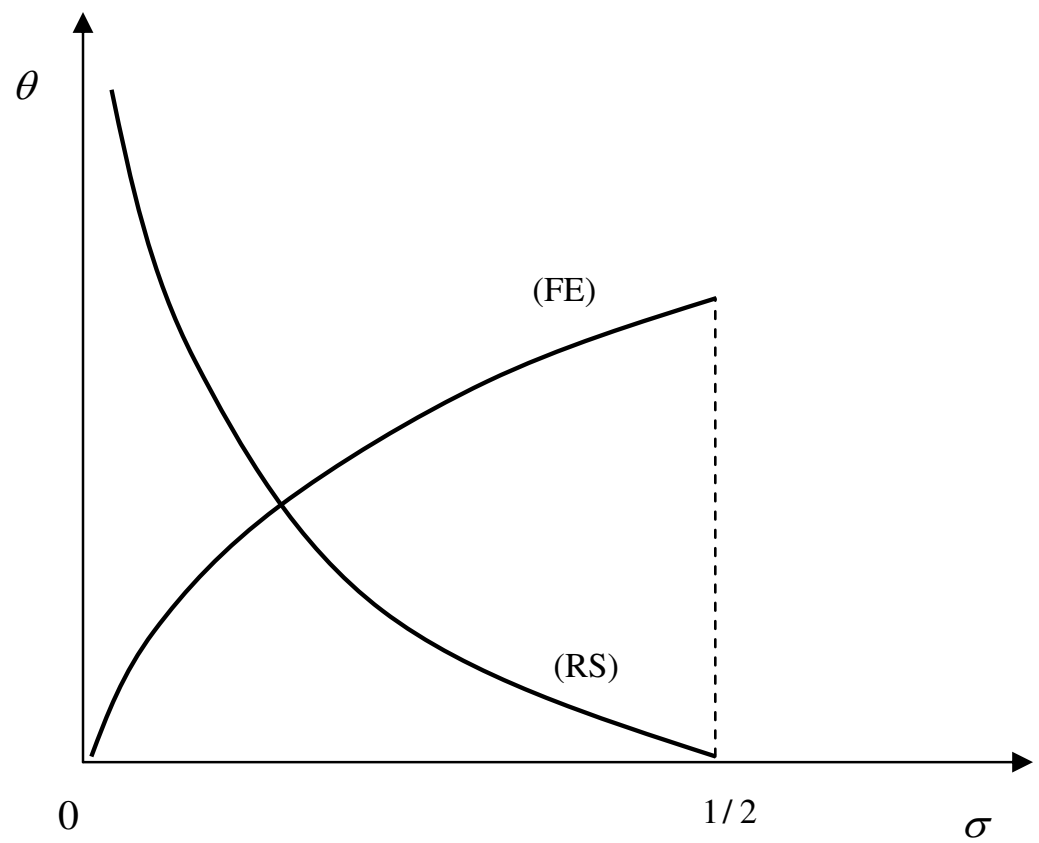

Fig.1 : Uniqueness of equilibrium when $y$ is concave 